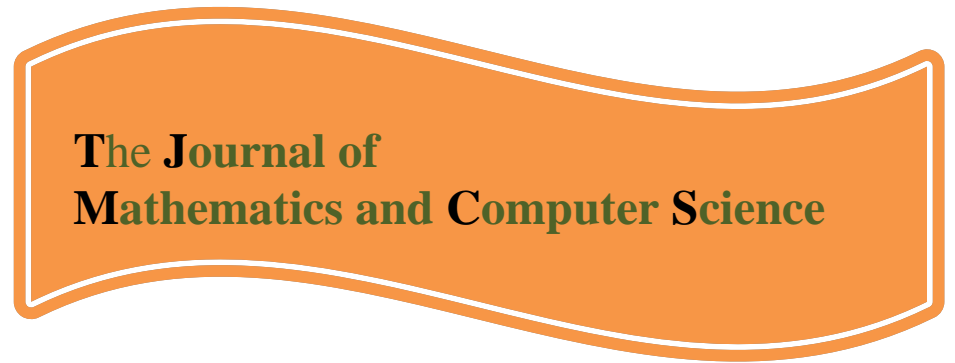

Available online at

\title{
http://www.TJMCS.com
}

The Journal of Mathematics and Computer Science Vol .2 No.1 (2011) 54-64

\section{A Genetic Fuzzy Approach for Building of Marketing Intelligence Systems for Consumer Behavior Modelling}

\author{
Hamid Reza Feili ${ }^{1 *}$, Reyhaneh Bijari ${ }^{2}$, Sepideh Zohoori ${ }^{3}$ \\ Hrfeili@gmail.com \\ R.bijari@gmail.com \\ Spdzohoori@gmail.com
}

Received: August 2010, Revised: November 2010

Online Publication: January 2011

\begin{abstract}
In this paper we debate on the causes of dissatisfactory of academic studies of marketing models. Next, we present a more complete methodology for knowledge discovery in data bases to be used in marketing causal modelling as a decision support tool in marketing management .This methodology is based on genetic fuzzy systems, a specific hybridization of artificial intelligence methods, that is proper for the problems we offer. Marketing intelligence system is called knowledge based marketing management support systems that is an avant-garde evolution in the use of KDD methods based on intelligence systems like this in our paper. The KDD process creates some basic questions for the professionals in this case that are completely discussed and solved next.After the theoretical presentation, this methodology is experimented on a consumer modelling application in interactive computer-mediated environments.
\end{abstract}

Keywords: Genetic Fuzzy Systems, Marketing Modelling, Consumer Behavior, Knowledge Discovery, Decision Support Systems.

\section{Introduction}

\footnotetext{
${ }^{*}$. Assistant Professor, Industrial Engineering, Alzahra University

2. B.S, Industrial Engineering, Alzahra University

${ }^{3}$. B.S, Industrial Engineering, Alzahra University
} 
In the past years 'information' has been increasingly contemplate a strategic resource whose adequate management has arisen as a key factor to explain entrepreneurial success (see, as e.g., Ein-Dor and Jones, 1985; Evans and Wurster, 1997; Laudon and Laudon,1998). The quantity and quality of relevant information that companies own when dealing with their decision-making processes has a fundamental role in ameliorating the degrees of uncertainty based on the business environment. Moreover, if we attend to the considerable turbulence and competitively defining the sectors in which companies operate, it is also essential to have timely information, obtained as a result of an efficient process (Hannula and Pirttimaki, 2003). The accomplishment of all these questions, and so, contributes to a reinforcement of both the firms' dynamicity and competitive response; of course, along with other factors related to the philosophy of marketing strategies, planning and execution that are beyond the research purposes tackle here.

It is easy to achieve a general agreement with the asseveration that the above ideas are the root cause of the appearance, improvement and evolution of Management Information Systems (MIS) in general, and Marketing Management Support Systems (MkMSS) in particular.

How to improve the impact and productivity of marketing actions addressed to customers has been and is especially now, or at least it should be, a constant concern of firms. This question explains, for instance, the transformation supported by the philosophy of the marketing function in the early 1980s, when the selling concept subjacent to the selling orientation evolved into the marketing concept characterizing the notorious market orientation. A advantageous market orientation necessarily needs that a firm worries about gathering and disseminating good and time-relevant market information to the different elemental parts of the organization (Hunt and Morgan, 1995; Kohli and Jaworski, 1990; Narver and Slater,1990).

Basically, firms looking to be market oriented should have information about their customers, competitors ,markets, and environment and utilize it when addressing their actions (Cravens et al., 2002). In this sense, as the amount of data to be accessed, stored and processed is usually so vast, firms necessarily have to make integrated use of information technologies and MkMSS to be competitive in the current information-intensive marketing contexts (Berthon and John, 2006). Moreover, inasmuch as it is expected that the market orientation philosophy evolves towards a more specific customer-centric way of managing the exchanges with markets (Kumar and Ramani,(2006), working with precise information and decision-support systems will become even more indispensable for the firm's survival. Specifically, this changing tendency should be better seen in the Web-based market contexts, where the medium's technology facilitates individualized interaction with customers (Hoffman and Novak, 1996), as well as access and facilities to obtain consumers' data (Lan, 2006; Smith, 2005). Consequently, the Web emerges as a very appealing data source for firms which, thanks to appropriate use of information technologies, may improve their knowledge about customers, thus fostering more value-added marketing actions and offers (see, as e.g., Aziz and Yasin, 2004; Wood, 2001).

In any case, regardless of the firms' market context, either the marketplace,

the market space or even both simultaneously, it seems evident that the main problem currently faced by market-oriented organisations is not the availability of information (data), but the possession of the necessary level of knowledge to take adequate decisions.

Moreover, not only the possession but also the use that firms make of such knowledge is said to be a notable factor in determining their competitive position (Silvi and Cuganesan, 2005). Currently, there is no doubt about the impact that a suitable management of knowledge must have on the firms' performance. In fact, Vargo and Lusch (2004, p.9), when proposing the set of foundational premises defining the new dominant logic of marketing, highlight knowledge as the underlying principle of "competitive advantage and economic growth and the key source of wealth".

This question explains why the analytical capabilities integrating the firm's MkMSS are so crucial for businesses in this moment of time. It is likely to expect that their variety and capabilities should have a relevant role in supporting the marketing managers' decisional processes, as they should allow the 
achievement of high value-added information about the customers' behavior. In this regard, it is been observed how the so-called knowledge-based MkMSS have increasingly taken a predominant position in the diversity of systems used by managers when supporting their decisions, from the middle 1980s to the present time (Shim et al., 2002; Wedel et al., 2000). These are usually based on methods for knowledge extraction from databases, which come from the computer science and artificial intelligence discipline; i.e., marketing systems based on, as e.g., artificial neural networks, genetic algorithms, fuzzy rules-based methods, etc. (Li et al., 2000; Wierenga and van Bruggen, 2000). Likewise, it is plausible to see an evolution, in the near future, towards the use of knowledge-based systems based on hybridizations - i.e., Soft Computing (SC) methodologies - of those artificial intelligence methods (Carlsson and Turban, 2002).

In sum, considering the previous paragraph as a backdrop, this paper introduces and empirically shows a knowledge discovery methodology to extract useful information patterns from consumers' databases, with a descriptive rule induction approach based on certain SC method (genetic-fuzzy systems). In this regard, we offer specific solutions to the diversity of questions related to such a Knowledge Discovery in Databases KDD process (e.g., how to process the original data, the design of the data mining state, the post-processing of the outputs, etc.). Hence, this is not a mere application of a data mining paradigm to a database. On the contrary, this research represents an important effort to adapt KDD solutions to the problem we face.

A knowledge discovery method based on genetic-fuzzy systems

Specifically, we make use of a supervised learning algorithm in the data mining process, as we work in the scenario that the marketing manager counts on the aprioristic information of a theoretical consumer behavior model. Basically, this drives the search process of the algorithm in the database. The practical experimentation of the methodology we propose is made by its application to an existing Webtrust consumer model, using a real database of internet users, which was originally estimated using Structural Equation Modelling (SEM). This initially allows us to show its potential to offer relevant pieces of information about the consumers being analyzed, in a linguistic format which is quite understandable and helpful for supporting the managers' decisions.

\section{What should we do with so much data that we have gathered?}

This question has become common in many organizations. The problem is that digitized information is easy to capture and fairly inexpensive to store. But why do people store so much data? It is easy and convenient to do and also people store data because they think some valuable assets are implicitly coded within it. In business, data captures information about critical markets, competitors, and customers.

Raw data is rarely of direct benefit. Its true value is predicated on the ability to extract information useful for decision support or exploration and understanding the phenomena governing the data source. Traditionally, analysis was a manual process. Some analysts familiar with the data provide summaries and generate reports. But nowadays, the amount of data is growing so fast that manual analysis (even if possible) simply cannot keep pace.

A community of researchers and practitioners interested in the problem of automating data analysis has grown steadily under the label knowledge discovery in databases (KDD) and data mining. The first KDD workshop was held in 1989; it has evolved into an annual international conference. The Second International Conference on Knowledge Discovery and Data Mining which attracted more than 500 attendees (Usam a Fayyadand Ramasamy Uthurusamy, 1996)

Statistics is at the heart of the problem of inference from data. Through both hypothesis validation and exploratory data analysis, statistical techniques are of fundamental importance. The article by Glymour, Madigan, Pregibon, and Smyth gives a statistical perspective, identifying a wealth of statistical results KDD can benefit from as well as some caveats and directions to consider.

In statistics, pattern recognition, and artificial intelligence (machine learning), algorithms are based on the assumption that data can be loaded into a computer's main memory. Wealth of interesting issues arises when the data is too large to fit in main memory. A perspective from databases, a field fundamental 
to KDD, is provided by Imielinski and Mannila, who identify challenges posed by KDD for database technology and postulate a new direction and view for both.

Quality of data is critical in data analysis. Inmon outlines the importance of and need for a data warehousing step in the KDD process. Issues specific to KDD applications in scientific data analysis are elaborated with illustrative examples by Fayyad, Haussler, and Stolorz. Etzioni explores the challenges and opportunities presented in discovering useful knowledge in the vast resources of the Internet, concluding that effective Web mining is feasible in practice.

Work addressing the core problems in KDD is ongoing; most problems of representation, search complexity, and use of prior knowledge to help search and statistical inference remain open and require serious attention. Nevertheless, successful applications continue to appear, driven mainly by the glut of database content that has clearly surpassed raw human processing abilities.

\section{Knowledge representation based on fuzzy rules}

Nowadays, one of the most successful tools to develop descriptive models is fuzzy modelling (Lindskog, 1997), sed to model a system making use of a descriptive language based on fuzzy logic with fuzzy predicates (Sugeno \& Yasukawa, 1993). The way to express fuzzy predicates is by means of IFTHEN rules with the following structure:

IF $A 1$ is $X 1$ and ... and An is Xn THEN B1 is Y1 and ... Bm is Ym

These rules set logical relationships among variables of a system by using qualitative values.

To properly work with this kind of qualitative valuations, linguistic variables (Zadeh, 1975) based on both Fuzzy Sets Theory and Fuzzy Logic (Zadeh, 1965) are used, so previous exemplified rule is known as a fuzzy rule. The use of fuzzy logic provides several benefits as: a higher generality, expressive power, ability to model real problems and, at last but not least, a methodology to exploit tolerance in the face of imprecision (Jorge Casillas, 2009)

In example, we can consider the linguistic variable age, which linguistic values could be teenager, young, adult, and old. Fuzzy rules can be considered as a knowledge extraction tool to discover intrinsic relationships contained in a database (Freitas, 2002). Thus, by means of fuzzy rules we can represent the relationship existing among different variables, thus deducing the patterns contained in the examined data. In knowledge discovery, the process to obtain these patterns must be automatic, or semi-automatic, discovered patterns must be comprehensible and they must provide useful information, and data must be invariably presented in substantial quantities (Witten \& Frank, 2000).

Useful patterns allow us to do non trivial predictions about new data. There are two extremes to express a pattern: like black boxes, whose internal behavior is incomprehensible; and like white boxes, whose construction reveals the pattern structure.

The difference lies in whether the generated patterns are represented with an easily examined structure, which can be used to reason and to inform further decisions. In other words, when the patterns are structured in a comprehensible way, they will be able to help in explaining something about the data. This trouble of KDD, the interpretability-accuracy trade-off, is also being currently faced in fuzzy modeling (Casillas et al., 2003a, 2003b) and will be posed in this proposal.

The use of fuzzy rules when developing the knowledge discovery process has some advantages as follows:

It become possible to use uncertainty data; they consider multi-variable relationships sufficiently; results are easily understandable by a human being; extra information can be easily added by an expert; the accuracy degrees can be easily modified to the problem necessity; and the process can be highly automatic with low human intervention.

Therefore, fuzzy logic will be used as a tool to structure the information of a consumer behavior model in a clear, legible, and close to the human being way. The interdependence of variables and the non-linear relationships among them can be shown by fuzzy system. 


\section{A marketing intelligent system for consumer behavior analysis}

This section introduces the process estimate consumer behavior based on fuzzy rules. It consists of preparing the data and of fixing the scheme followed to represent the knowledge existing in the data. Once defined these aspects, a machine learning method based on GAs is pro- posed to automatically extract fuzzy models.

\subsection{Data gathering}

We should collect the relevant data to the variables stating the theoretic consumer behavior model of reference .Data are obtained traditionally by means of questionnaires in marketing .Therefore, attention should be paid to how consumer behavior modelers face and develop the measurement process of variables (as latent/unobserved) complex behavioral models contain.

Knowledge Discovery by Genetic Fuzzy Systems applied to Consumer Behavior Modelling point of the KDD process is used to give suitable and adapted solutions to the specific data found in consumer behavior modeling.

Measuring streams for these latent variables in marketing modeling can be classified into two groups depending on if they state that these constructs can or cannot be perfectly measured by means of observed variables (indicators). Though consumer behavior modelers tended to make use in the beginning of what was known as the operational definition philosophy, a more convenient and reasonable position is that ulteriorly based on the partial interpretation philosophy which distinguished between variables .This latter approach of measurement, being currently prevailing in the marketing modeling discipline, poses to collectively consider multiple indicators - imperfect when considered individually, though reliable when considered altogether- of the subjacent construct to obtain valid measures (Steenkamp\& Baumgartner, 2000) .

\subsection{Data Processing}

It is essential to conform the collected data to a scheme easily tractable by fuzzy rule learning methods .The approach should be aware of the special features of the available data (with several items or indicators to describe a specific variable) when conforming the observed variables to a fuzzy rule learning method.

An intuitive approach could directly reduce the items of certain variables to a single value (e.g., by arithmetic mean). Another possibility would be to expand any multi-item example (the result of a questionnaire filled out by a consumer) to several single-item examples and, subsequently, reduce the data size with some instance of selection process (Casillas, Martinez-Lopez, 2004).

The problem of these approaches is that the data must be transformed, so relevant information may be lost.We propose a more sophisticated process that allows working with the original format without any pre-processing stage :the multi-item fuzzification.

Thus, a T-conorm operator (e.g., maximum ), traditionally used in fuzzy logic to develop the union of fuzzy sets, is applied to accumulate the partial information given by each item during the inference process.

\subsection{Representation and Inclusion of Expert Knowledge}

There are some issues should be considered in this step: modeling the set of variables, the transformation of marketing scales (used for measuring such variables) into fuzzy semantic and the fuzzy rule structure. We represent some approaches to fix these components. They are based on the marketing expert's capability to express the knowledge in a humanly understandable format by fuzzy logic. 


\subsubsection{Fuzzy Semantics from Expert Knowledge}

When the marketing modeler has determined the theoretical constructs and the observed variables, a transformation of the original marketing scales used for measuring those observed variables into linguistic terms should be done. With the aim of simplifying the problem, we focus on Likert-type, differential semantic and rating scales used in these models. The transformation should be practiced taking into account three main questions:

1 .The number of linguistic terms to be used for each variable must be defined.

In this case it is useful to linguistically express the "medium" or "unconcerned" concept, so an odd number seems to be a good approach. Since traditional interval scales used in marketing usually present between 5 to 9 different degrees, the use of three or five linguistic terms (fuzzy sets) is enough to map these values.

2 .The membership function type stating the behavior of certain fuzzy variables should be also defined. We pose that it is more appropriate to use linear functions, in as much as it facilitates the latter interpretation of relations.

3 .The membership function shapes should also be fixed. It is proposed to impose some properties in order to ensure good interpretability .Extreme values of the interval should have a membership degree 1 to extreme labels. Mean value of the interval should have membership 1 to medium label .Likewise, it is considered strong Ruspini's fuzzy semantics (the sum of the membership degrees of every value to the set of linguistic terms is 1 ) in order to ensure good interpretability .Finally, in order to statistically unbias the significance of every linguistic term, it is imposed the same covering degree .Thus, the membership function shapes where, given the set $S=\{\min , \ldots, \max \}$ defining the interval, is defined and they hold the following condition:

$$
\sum_{k \in S} \mu_{A_{i}}(\mathrm{k})=\frac{\max -\min }{l}, \forall A_{i} \in A
$$

$\mathrm{l}=$ the number of linguistic terms and $\mathrm{A}=\{\mathrm{A} 1, \ldots \mathrm{Al}\}$ the set of them

In this section an example will be posed based on the transformation of a seven-point rating scale (a typical marketing scale used to measure the observed variables/indicators related to certain construct) into a fuzzy semantic which be included the three linguistic terms Low, Medium, and High represented by the means of corresponding fuzzy sets characterized by the following three membership function:

$$
\mu_{\text {Low }}(\mathrm{x})=\left\{\begin{array}{c}
\frac{4-x}{3} \text { if } 2 \leq x \leq 4 \\
0 \quad \text { otherwise }
\end{array} \quad \mu_{\text {Medium }}(\mathrm{x})=\left\{\begin{array}{c}
\frac{\mathrm{x}-1}{3} \text { if } 1 \leq \mathrm{x} \leq 4 \\
\frac{7-\mathrm{x}}{3} \text { if } 4<x \leq 7 \\
0 \quad \text { otherwise }
\end{array} \mu_{\mathrm{High}}(\mathrm{x})=\left\{\begin{array}{cc}
\frac{7-x}{3} & \text { if } 5 \leq x \leq 8 \\
0 & \text { otherwise }
\end{array}\right.\right.\right.
$$

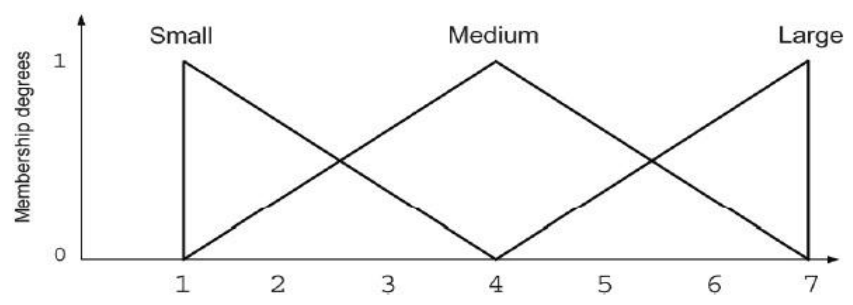

Fig1. Transformation of a seven-point rating scale into a three-triangular fuzzy semantic

These rules can show many relationships among the variables; however, all of them will not match the existing data exactly .So, some measures will be required to assess the quality of each rule with 
respect to the data.These measures can be considered a kind of statistical computation .In this paper, it will be considered two important values :support and confidence .On the one hand, support (whose real value is in $[0,1]$ will give us an idea about in which degree the rule represents the cases of the data set . For example, a support of 0.25 could be understood as the rule that covers $25 \%$ of the available cases.It will be interested in obtaining fuzzy rules with a support as high as possible since the rule will be more general and will represent a higher portion of the sample .On the other hand, confidence ( whose real value is also in $[0,1]$ ), shows how accurate the fuzzy rule is .Since the fuzzy rule predicts a relationship between the antecedent and the consequent, it is needed to know in which degree such a prediction appears in the available data set .For example, if a fuzzy rule has a confidence of 0.9 , we can say that, according to the available data, the fuzzy rule is $90 \%$ true .Of course, we are interested in obtaining fuzzy rules with a high degree of confidence.

As one can imagine, support and confidence are two contradictory features .Inasmuch as the degree of representation is higher, it is more difficult to accurately express the relationships among variables . One fuzzy rule will be clearly preferable to another if the former has higher values of both support and confidence.

In the following, it will be shown some examples of fuzzy rules and the computation of the corresponding support and confidence values from the data set.

\section{R1: If quality is low and on time delivery is medium then price is medium.}

$$
\begin{aligned}
& \mu_{\text {Low }}\left(\mathrm{x}_{1}^{(1)}\right)=\max \left\{\mu_{\text {Low }}(1), \mu_{\text {Low }}(2), \mu_{\text {Low }}(1)\right\}=\max \{1,0.67,1\}=1 \\
& \mu_{\text {Medium }}\left(x_{2}^{(1)}\right)=\max \left\{\mu_{\text {Medium }}(5), \mu_{\text {Medium }}(6)\right\}=\max \{0.67,0.33\}=0.67 \\
& \mu_{\mathrm{A}^{(1)}}\left(\mathrm{x}^{(1)}\right)=\min \left\{\mu_{\text {Low }}\left(\mathrm{x}_{1}^{(1)}\right), \mu_{\text {Medium }}\left(\mathrm{x}_{2}^{(1)}\right), \mu_{\text {Low }}(1)\right\}=\min \{1,0.67\}=0.67 \\
& \mu_{\text {Medium }}\left(y^{(1)}\right)=\max \left\{\mu_{\text {Medium }}(1), \mu_{\text {Medium }}(2)\right\}=\max \{0,0.33\}=0.33 \\
& \mu_{\text {Low }}\left(\mathrm{x}_{1}^{(2)}\right)=0, \mu_{\text {Medium }}\left(\mathrm{x}_{2}^{(2)}\right)=0.33, \mu_{\mathrm{A}^{(1)}}\left(\mathrm{x}^{(2)}\right)=0, \mu_{\text {Medium }}\left(y^{(2)}\right)=0.33 \\
& \mu_{\text {Low }}\left(\mathrm{x}_{1}^{(3)}\right)=0, \mu_{\text {Medium }}\left(\mathrm{x}_{2}^{(3)}\right)=0.33, \mu_{\mathrm{A}^{(1)}}\left(\mathrm{x}^{(3)}\right)=0, \mu_{\text {Medium }}\left(y^{(3)}\right)=0 \\
& \mu_{\text {Low }}\left(\mathrm{x}_{1}^{(4)}\right)=0, \mu_{\text {Medium }}\left(\mathrm{x}_{2}^{(4)}\right)=0.67, \mu_{\mathrm{A}^{(1)}}\left(\mathrm{x}^{(4)}\right)=0, \mu_{\text {Medium }}\left(y^{(4)}\right)=0.67 \\
& \quad \operatorname{Support}\left(R_{1}\right)=\frac{0.67 .0 .33+0+0+0}{4}=0.05556 \\
& \text { Confidence }\left(R_{1}\right)=\frac{0.67 . \max \{1-0.67 .0 .33\}+0+0+0}{0.67+0+0+0}=0.33333
\end{aligned}
$$

\section{R2: If quality is medium and on time delivery is medium then price is medium.}

$$
\operatorname{Support}\left(R_{2}\right)=0.13889 \quad \text { Confidence }\left(R_{2}\right)=0.44445
$$

\section{R3: If quality is medium and on time delivery is low then the price is medium.}

$$
\operatorname{Support}\left(R_{3}\right)=0.16667 \quad \text { Confidence }\left(R_{2}\right)=0.66667
$$

This third rule includes two linguistic terms in the variable conservatism. With it, the support is higher since we can cover the data set to a higher degree compared to rule R2 (it is obvious since R3 is 
more general than R2). Also, the confidence is improved; therefore the third rule is clearly better than the previous ones.

\subsubsection{Input/Output Linguistic Variables from Expert Knowledge}

When the marketing expert fix the structure of the model under the base of the theoretic model, fuzzy rules are used to relate input (antecedents) with output (consequents)variables. hypotheses contained in the model can be directly used to define IF-THEN structures by considering the dependencies shown among the variables.Thus, a fuzzy rule base obtained for each consequent( endogenous construct )considered and its respective set of antecedents.

\section{Machine Learning (Data Mining Process)}

\subsection{Fuzzy Rule Structure}

In data mining, using a learning process with a high degree of Interpretability is very difficult. So, it would be opted for a compact description based on the disjunctive normal form (DNF).This fuzzy rule structure has the following form:

where each input variable Xi takes as a value a set of linguistic terms $\widetilde{A}_{i}=\left\{A_{i 1}, \ldots, A_{i n_{i}}\right\}$, whose members are joined by a disjunctive (T-conorm ) operator, whilst the output variables $Y_{J}, J \in\{1, \ldots, m\}$ remains a usual linguistic variable with a single label associated.We use the bounded sum $\min \{1, \mathrm{a}+\mathrm{b}\}$ as $\mathrm{T}$-conorm .The structure is a natural support to allow the absence of some input variables in each rule.

\subsection{Multi-item fuzzification}

[

It is proposed that an extension of the membership degree computation, to consider the set of items available for each input/output variable the so-called multi-item fuzzification. The process is based on a union of the partial information provided by each item. We have Xi and Yj measured by the vectors of items

$$
\begin{aligned}
& x_{i}=\left(x_{1}^{(i)}, \ldots, x_{h_{i}}^{(i)}, \ldots, x_{p_{i}}^{(i)}\right) \\
& y_{j}=\left(y_{1}^{(j)}, \ldots, y_{t_{i}}^{(j)}, \ldots, y_{q_{i}}^{(j)}\right)
\end{aligned}
$$

And the fuzzy proposition $X_{i}$ is $\tilde{A}_{i}$ is computed as follows:

$\mu_{\tilde{A}_{i}}(\mathrm{x})=\max _{h_{i}=\left\{1, \ldots, p_{i}\right\}} \min \left\{1, \sum_{k=1}^{n_{i}} \mu_{A_{i k}}(x)\right\}$

While the fuzzy proposition $Y_{j}$ is $B_{j}$ is computed as follows:

$$
\mu_{\widetilde{B}_{i}}\left(y_{j}\right)=\max _{\mathrm{k}_{\mathrm{j}} \in\left\{1, \ldots, \mathrm{m}_{\mathrm{j}}\right\}} \mu_{B_{j}}\left(y_{k_{j}}^{j}\right)
$$

\subsection{Discovery process}

The subgroup discovery technique (Lavrac et al., 2004)is used in this proposal. 
Hamid Reza Feili, Reyhaneh Bijari, Sepideh Zohoori/ TJMCS Vol .2 No.1 (2011) 54-64

It involves running the genetic algorithm once for each possible consequent combination, extracting the most interesting groups (antecedent combination) for each of them. The use of a multi-objective approach allows the algorithm to extract rules with different properties, according to two different criteria as described here.

\subsection{Multi-objective genetic algorithm}

The proposed multi-objective genetic algorithm consists of the following components:

- Coding scheme: Each individual of the population represents a single fuzzy rule.

Since only the antecedent must be encoded (because the consequent is fixed for each run), a binary representation may be used. Thus, the coding scheme size is equal to the sum of the number of linguistic terms used in each input variable.

The allele ' 1 ' means that the corresponding linguistic term is used in the corresponding variable. For example, assuming we have two input variables and three linguistic terms (small, medium, and large) for each of them, the fuzzy antecedent [ IF $X 1$ is Small and $X 2$ is \{Medium or Large\}] is encoded as [100|011].

- Evolutionary scheme: A generational approach with the multi-objective NSGA-II replacement strategy (Deb et al., 2002) is considered.

- Criteria: We employ two different objectives to extract fuzzy rules with different characteristics:

Support: This objective function measures the representation degree of the corresponding fuzzy rule among the available data. It is computed by gathering the matching of all the analyzed data to the fuzzy rule antecedent as follows:

$$
\operatorname{Support}(R)=\frac{1}{N} \sum_{e=1}^{N} \mu_{A}\left(x^{(e)}\right) \cdot \mu_{B}\left(y^{(e)}\right)
$$

with $N$ as the data set size.

Confidence: This objective function measures the quality (accuracy) of the relationship between input and output variables described by the analyzed fuzzy rule. It is computed as follows:

$$
\operatorname{Conffidence}(R)=\frac{\sum_{e=1}^{N}\left(\mu_{A}\left(x^{(e)}\right) \cdot \max \left\{1-\mu_{A}\left(x^{(e)}\right), \mu_{B}\left(y^{(e)}\right)\right\}\right)}{\sum_{e=1}^{N} \mu_{A}\left(x^{(e)}\right)}
$$

\subsection{Genetic operators}

The initial population is built defining so many groups (equal in size)as there are different consequents .In each of them, chromosomes are generated fixing such consequents and randomly building a simple antecedent where each input variable is related to a linguistic term .The two operators of reproduction only act in the part of the antecedent of the rule. This fact ensures that the size of every subgroup in the population is constant .In this way, we allow the algorithm to independently explore, but simultaneously, each group.

It will be employed a multipoint crossover operator which selects two crossover points (in the part of the antecedent) and interchanges the central sub-chain. The operator of mutation randomly selects a variable of the antecedent of the fuzzy rule coded in the chromosome and carries out some of the three following operations :expansion, which flips to 1 a gene of the selected variable; contraction, which flips to 0 a gene of the selected variable; or shift, which flips to 0 a gene of the variable and flips to 1 the gene immediately before or after it.The selection of one of these mechanisms is made randomly among the available choices (e.g., contraction cannot be applied if only a gene of the selected variable has the allele 1) 


\section{Case study}

In this paper we used this methodology in cement industry, and show that it can be useful for other industries.

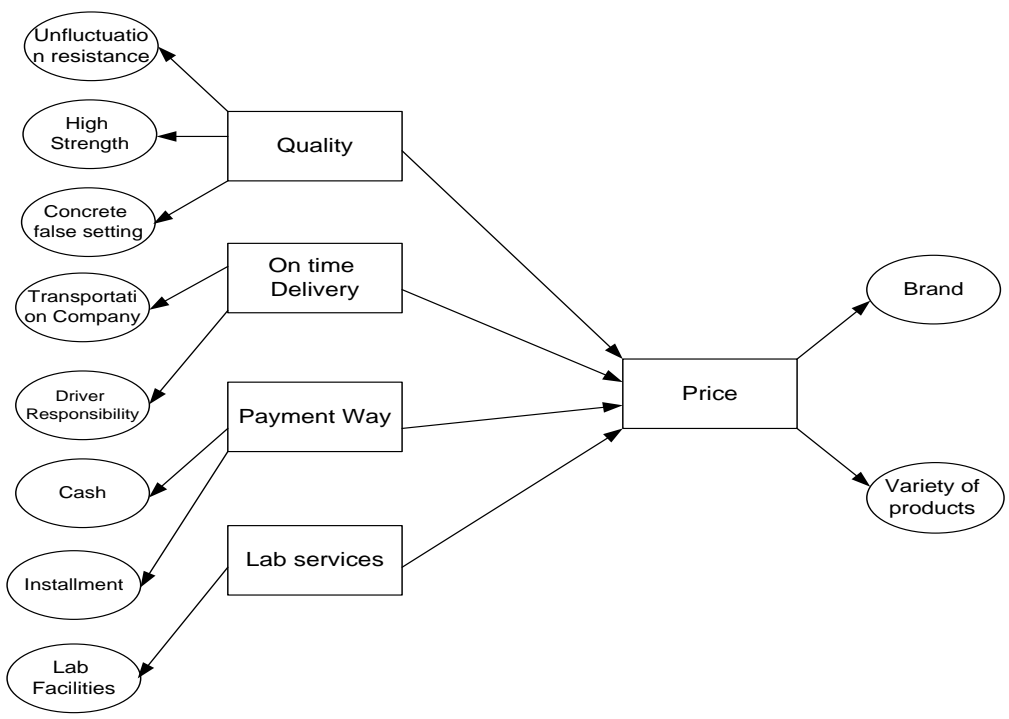

Fig2. Consumer behavior model used for the experimentation

The first step is defining the rules as follows:

$\mathrm{R}_{1}=$ If Quality is HIGH ,On time delivery is HIGH, Lab services are HIGH and Payment Way is LOW then Price is HIGH.

$\mathrm{R}_{2}=$ If Quality is HIGH ,Lab services are HIGH and Payment Way is LOW then Price is HIGH.

$\mathrm{R}_{3}=$ If Quality is HIGH ,On time delivery is HIGH or MEDIUM ,Lab services are HIGH and then Price is $\mathrm{HIGH}$.

$\mathrm{R}_{4}=$ If Quality is HIGH ,Lab services are MEDIUM or HIGH and Payment Way is LOW or MEDIUM then Price

HIGH.

Second, we should compute support and confidence.so we do it and show them in the follow table:

Table1.Pareto set of fuzzy rules that relate Quality, On time delivery, Lab services and Payment Way with Price

\begin{tabular}{|c|c|c|c|c|c|c|c|c|c|c|c|c|c|c|c|c|c|}
\hline & \multicolumn{3}{|c|}{ Quality } & \multicolumn{3}{|c|}{$\begin{array}{l}\text { On time } \\
\text { delivery }\end{array}$} & \multicolumn{3}{|c|}{$\begin{array}{l}\text { Lab } \\
\text { Services }\end{array}$} & \multicolumn{2}{|c|}{$\begin{array}{l}\text { Payment } \\
\text { Way }\end{array}$} & & \multicolumn{3}{|c|}{ Price } & \multirow{2}{*}{$\begin{array}{l}\text { Support } \\
0.011838\end{array}$} & \multirow{2}{*}{$\begin{array}{l}\text { Confidence } \\
0.938272 \\
\end{array}$} \\
\hline $\mathrm{R}_{1}$ & - & - & $\mathrm{H}$ & - & - & $\mathrm{H}$ & - & - & $\mathrm{H}$ & $\mathrm{L}$ & - & - & - & - & $\mathrm{H}$ & & \\
\hline $\mathrm{R}_{3}$ & - & - & $\mathrm{H}$ & - & M & $\mathrm{H}$ & - & - & $\mathrm{H}$ & - & - & - & - & - & $\mathrm{H}$ & 0.131344 & 0.906223 \\
\hline $\mathrm{R}_{4}$ & - & - & $\mathrm{H}$ & - & - & - & - & M & $\mathrm{H}$ & $\mathrm{L}$ & M & - & - & - & $\mathrm{H}$ & 0.298309 & 0.872153 \\
\hline $\mathrm{R}_{7}$ & - & M & $\mathrm{H}$ & - & - & - & - & - & - & - & - & - & - & - & $\mathrm{H}$ & 0.778952 & 0.790380 \\
\hline
\end{tabular}




\section{Hamid Reza Feili, Reyhaneh Bijari, Sepideh Zohoori/ TJMCS Vol .2 No.1 (2011) 54-64}

We have practiced a multi-objective optimization in order to obtain rules with high degree of support and confidence.

\section{Concluding remarks}

There is an interesting problem with KDD in relation to marketing causal modelling and its resolution by Genetic Fuzzy Systems.The problem presents a specific type of uncertain data that justifies the use of fuzzy rules and with multi-objective optimization, we obtain rules with high degrees of support and confidence .The KDD methodology proposed is successfully applied to a real problem of consumer behavior modeling in online environments, where we have offered an overall perspective of how it works . The results we have obtained have been satisfactory .Summarizing, we believe that its use is very promising for academic and, especially, managerial purposes.

\section{References}

[1]Akhter, F., Hobbs, D., \& Maamar, Z. (2005). A fuzzy logic-based system for assessing the level of business-toconsumer (B2C) trust in electronic commerce. Expert Systems with Applications, 28(4), 623-628.

[2]Baumgartner, H., \& Homburg, C. (1996). Applications of structural equations modelling in marketing and consumer research: A review. International Journal of Research in Marketing, 13, 139-161.

[3] Jorge Casillas, Francisco J. Martı́nez-Lo'pez. Mining uncertain data with multiobjective genetic fuzzy systems to be applied in consumer behaviour modelling(2009)

[4]Casey, C., \& Murphy, C. (1994). Expert system in marketing: An application for pricing new products. Expert Systems with Applications.552-545,(4)4,

[5]Casillas, J., Cordo' n, 0., del Jesus, M. J., \& Herrera, F. (2005). Genetic tuning of fuzzy rule deep structures preserving interpretability and its interaction with fuzzy rule set reduction. IEEE Transactions on Fuzzy Systems, 13(1), $13-29$.

[6]Casillas, J., Martı́nez-Lo'pez, F. J., \& Martı́nez, F. J. (2004). Fuzzy association rules for estimating consumer behaviour models and its application to explain trust in Internet shopping. Fuzzy Economic Review, IX(2), 3-26.

[7] Beynon, M., Curry, B., \& Morgan, P. (2001). Knowledge discovery in marketing. An approach through rough set theory. European Journal of Marketing, 35(7/8), 915-935.

[8]Cordo'n, O., Herrera, F., Hoffmann, F., \& Magdalena, L. (2001). Genetic fuzzy systems: Evolutionary tuning and learning of fuzzy knowledge bases. Singapore: World Scientific.

[9]Csikszentmihalyi, M. (1975). Play and intrinsic rewards. Journal of Humanistic Psychology, 15(3), 41-63.

[10]Csikszentmihalyi, M. (1977). Beyond boredom and anxiety (2nd ed.). San Francisco: Jossey-Bass.

[11]Gatignon, H. (2000). Commentary on Peter Leeflang and Dick Wittink's ''Building models form marketing decisions: past, present and future. “

[12]Hurley, S., Moutinho, L., \& Stephens, N. M. (1995). Solving marketing optimization problems using genetic algorithms. European Journal of Marketing, 29(4), 39-56.

[13]Ha, S. H., \& Park, S. C. (1998). Application of data mining tools to hotel data mart on the Intranet for database marketing. Expert Systems with Applications, 15(1), 1-31.

[14]Hoffman, D., \& Novak, T. (1996). Marketing in hypermedia computermediated environments: Conceptual foundations. Journal of Marketing, 60(July).

[15]Fayyad, U. M., \& Simoudis, E. (1995). Knowledge discovery and data mining: Tutorial. In Proceedings of the 14 th international joint conference on artificial intelligence. Montreal, Canada.

[16]Fish, K. E., Johnson, J. D., Dorsey, R. E., \& Blodgett, J. G. (2004). Using an artificial neural network trained with a genetic algorithm to model brand share. Journal of Business Research, 57(1).

[17]International Journal of Research in Marketing, 17, 209-214 (Special issue on marketing modelling on the threshold of the 21st century). 